\title{
Editorial
}

\section{Alfabetización de datos}

DOI: 10.29236/sistemas.n152a1

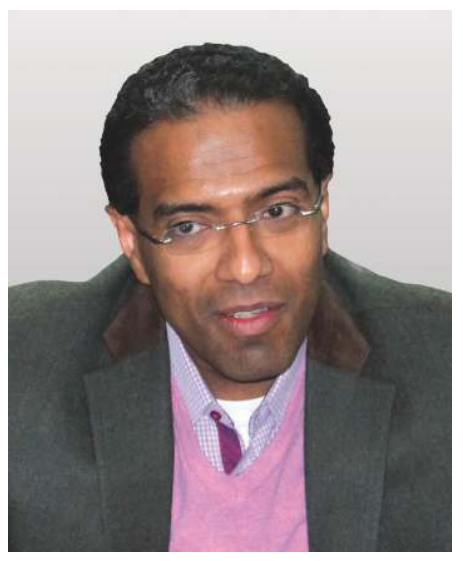

Un reto para los profesionales del siglo XXI.

\section{Jeimy J. Cano M.}

Los datos representan ahora la nueva moneda en el contexto digital y establecen realidades inéditas, además de revelar patrones antes desapercibidos, como fuente de oportunidades y retos para las personas y las empresas. Por tan- to, desarrollar la competencia para su gestión y tratamiento se convierte en un imperativo para los profesionales del siglo XXI que quieren alcanzar posiciones estratégicas en un escenario cada vez más incierto e inestable. 
Por lo anterior, la Asociación Colombiana de Ingenieros de Sistemas -ACIS-, ha dedicado este número de la revista Sistemas a explorar y revisar el reto de la alfabetización de datos, como ventana emergente de oportunidades y desafíos que la sociedad contemporánea enfrenta.

En esa dirección, ha convocado a destacados profesionales para que compartan sus reflexiones y análisis sobre esta temática que resulta de interés personal, profesional y empresarial.

En primer lugar, Eduardo Escallón, decano de la Facultad de Educación de la Universidad de Los Andes nos introduce en las tensiones de los currículos y los conocimientos computacionales como elementos claves en el escenario de los grandes datos, y la necesidad del desarrollo de un pensamiento crítico para concretar la potencialidad de la información y poder proponer soluciones constructivas que mejoren el sistema educativo y la sociedad en general.

Le sigue la entrevista realizada a la ministra de tecnologías de información y comunicaciones, Sylvia Constaín Rengifo, quien desde la mirada del ciudadano espera una acelerada apropiación de las tecnologías de información, con el fin de que se planteen soluciones a sus problemáticas cotidianas y se generen propuestas interesantes que impacten los diversos sectores de la economía, apuesta que se traduce en una amplia agenda de iniciativas.

En la sección Cara y sello están las reflexiones de los invitados al tradicional foro de la revista, cuyo tema central giró alrededor de la pregunta ¿somos analfabetas de datos? Los participantes de la industria y la academia compartieron diferentes puntos de vista, insistiendo en la necesidad del desarrollo de habilidades y aptitudes para el tratamiento de los datos, orientado a motivar la construcción de conocimiento para la transformación de las organizaciones, los negocios y la sociedad.

La investigación realizada para este número es un ejercicio de exploración sobre el estado de la alfabetización de datos en el gremio de los profesionales de tecnología de información. Los resultados muestran tendencias marcadas alrededor del uso de herramientas analíticas básicas y avanzadas, visualización de los análisis, integridad de las fuentes y protección de los datos personales. Si bien no es un estudio concluyente, sí establece una base de discusión para una temática en desarrollo.

Finalmente, el contenido también contempla un artículo realizado por un grupo de investigadores de la Universidad de los Andes, relacionado con la brecha digital y el desarrollo de habilidades y competencias que permitan habilitar un cam- 
bio social e inclusivo, particularmente desde las regiones.

Como se puede observar, la temática tiene diversas aristas, puntos de encuentro y desencuentro, propuestas locales e internacionales; es decir, una dinámica particular que cada vez más encuentra inte- resados en avanzar en la construcción de referentes académicos y empresariales hacia una alfabetización de datos, para consolidar a futuro una cultura organizacional y social como fundamento para la toma de decisiones y apalancadora de un proyecto de vida.

Jeimy J. Cano M., Ph.D, CFE, CICA. Ingeniero y Magíster en Ingeniería de Sistemas y Computación por la Universidad de los Andes. Especialista en Derecho Disciplinario por la Universidad Externado de Colombia. Ph.D en Business Administration por Newport University, CA. USA. y Ph.D en Educación por la Universidad Santo Tomás. Profesional certificado como Certified Fraud Examiner (CFE), por la Association of Certified Fraud Examiners y Certified Internal Control Auditor (CICA) por The Institute of Internal Controls. Es director de la Revista Sistemas de la Asociación Colombiana de Ingenieros de Sistemas-ACIS-. 